\title{
A Review of Coleoptera Diversity of Chhattisgarh: Updated Checklist 2015
}

\author{
Namita Gajendra, S. K. Prasad \\ School of Studies in Bioscience (Life Sciences), Pt. Ravishankar Shukla University, Raipur-492010, Chhattisgarh, India
}

\begin{abstract}
Chhattisgarh is enriched in diversity of flora and fauna. It is spread from $80^{\circ} 15^{\prime}$ to $84^{\circ} 24^{\prime} \mathrm{E}$ and $17^{\circ} 46^{\prime}$ to $24^{\circ} 5 \mathrm{~N}$. Chhattisgarh state is covered by dense forest and hills. The present review documented several species of Coleoptera order in Chhattisgarh. There are 194 species of beetles belonging to 6 families and 23 subfamilies of order Coleoptera (refer checklist), documented from Chhattisgarh. The families viz. Scarabaeidae (78 species), Tenebrionidae (12 species), Geotrupidae (4 species), Cerambycidae (3 species) and Hybosoridae (2 species). Chrysomelidae family indicates highest number of species (95 species).
\end{abstract}

Keywords: Coleoptera, diversity, Chhattisgarh, beetles, checklist

\section{Introduction}

Chhattisgarh is endowed with diverse groups of flora and fauna. Geographical location of Chhattisgarh state is in between $80^{\circ} 15^{\prime}$ to $84^{\circ} 24^{\prime} \mathrm{E}$ and $17^{\circ} 46^{\prime}$ to $24^{\circ} 5 \mathrm{~N}$. It is covered by dense forest and hills. The present review on paper is several species of Coleoptera order in Chhattisgarh. Coleoptera is one of the largest orders of class insect and they are cosmopolitan in distribution. Overall 3,50,000 species of beetles are identified worldwide (Choate, 2001) and about 1,5088 species are found in India (Kazmi and Ramamurthy, 2004). The diversity of beetles is extremely broad. Beetles are particular species that are adapted to almost every type of diet. Coleoptera are minute to very large insect of various form and colour but mostly powerfully sclerotized forewings adapted into defensive covers or elytra. The compound eyes are normally prominent (Thakare et al. 2012). Coleoptera comprise a speciose group and there are conspicuous component of the beetle fauna of the world. Beetles are debris feeder, some feed on plants by making hole into plant tissue and tunnels in wood or under bark, fungi, dung, flesh, flower, fruit, leaves and pollen while some beetles are predator, thus play an important role in nature's sanitation. ( (Pawara et al., 2014). Scarabaeidae family is the largest family of insect which includes more than 30000 species in the world. Dung beetles, that family of Scarabaeide have been effectively used to decrease the population of parasitic worms and pestilent flies that breed in cattle. Ground beetles (Carabidae) are common predators of many diverse insects and other arthropods, including wireworms, fly eggs, caterpillars, and other (Banerjee, 2014). Leaf beetles (Chrysomelidae) being phytophagous, the group includes various established and potential agricultural pests.

\section{Literature Survey}

Kalaichelvan and Verma (2005a) explained in detail about life cycle of Chrysomelidae: Criocerinae from Bhilai-Durg. Chandra and Gupta (2012) investigated 4 species of the genus Bolbohamatum; B. phallosum Krikken, 1980, B. laterale (Westwood, 1848), B. marginale Krikken, 1980 and B. calanus (Westwood 1848), and one species of genus Bolbogonium; B. insidiosum Krikken, 1977 were studied from the states Madhya Pradesh and Chhattisgarh. $B$. calanus constitutes new reports for Chhattisgarh. Chandra and Gupta (2013) investigated 43 species of scarab beetles belonging to 25 genera, 16 tribes and 8 subfamilies in two families Scarabaeidae and Hybosoridae of the superfamily Scarabaeoidae from Barnawapara wildlife sanctuary, Chhattishgarh. The dominant subfamily scarabaeinae includes 31 species followed by subfamilies Aphodiinae, Cetoniinae and Hybosorinae consisting of a single species each then Melolonthinae with three species Orphninae, Dynastinae and Rutelinae with two species each. Chandra and Gupta (2013) reported taxonomic account of 52 species of dung beetles belonging to 22 genera, 12 tribes, 04 subtribes, 5 subfamilies and 03 families viz. Scarabaeidae, Hybosoridae and Geotrupidae from chhattishgarh. The maximum number of species were collected from Barnawapara Wildlife Sanctuary (41 species) followed by Achanakmar-Amarkantak Biosphere Reserve (27 species) and Kanger valley National Park (15 species). Total 95 species collected by Kalaichelvan and Verma (2005b) belonging to the subfamilies criocerinae (10 species), Chlamisinae (1 species), Clytrinae (6 species), Eumolpinae (6 species), Cassidinae(18 species), Cryptocephalinae (8 species), Chrysomelinae(4 species), Hispinae (4 species), and Galerucinae(18 species) of the chrysomelid fauna of Bhilai-Durg. Chandra and Gupta (2012) collected 581 specimens of the scarab beetles from AchanakmarAmarkantak Biosphere reserve which yielded the identification of 52 species belonging to 24 genera and 05 subfamily Rutelinae, Cetoniinae, Scarabaeinae, Dynastinae and Melolonthinae of family Scarabaeidae. Chandra and Gupta (2012) studied diversity and relative abundance of pleurostict scarabaeidae (Coleoptera: Dynastinae, Scarabaeidae, Rutelinae, Cetoniinae, Melolonthinae) in Achanakmar-Amarkantak Biosphere reserve, Chhattishgarh. 12 new records of darkling beetles to 07 districts of Chhattisgarh state viz;Bilaspur, Raigarh, Koriya, Surguja, Durg, Raipur and Bastar. The recorded 12 species belonging to 09 genera of 05 tribes under 03 subfamilies of the family Tenebrionidae (Coleoptera) by Hegde et al (2015).

\section{Conclusion}

Chhattisgarh provides diverse atmosphere to different species of coleopteran. A checklist (Table 1) of the reported 


\section{International Journal of Science and Research (IJSR) \\ ISSN (Online): 2319-7064 \\ Index Copernicus Value (2013): 6.14 | Impact Factor (2015): 6.391}

Coleoptera fauna from different localities of Chhattisgarh state; there are 194 species of beetles belonging to 6 families and 23 subfamilies of order Coleoptera (refer checklist), documented from state Chhattisgarh. The families Viz. Scarabaeidae, Hybosoridae, Geotrupidae, Cerambycidae, Chrysomelidae, Tenebrionidae. The highest numbers of beetles were collected from the family Chrysomelidae (95 species belonging to 10 subfamily) followed by
Scarabaeidae (78 species belonging to 7 subfamily), Tenebrionidae (12 species under the 3 subfamily), Geotrupidae (4 species belonging to 1 subfamily), Cerambycidae (3 species under the 1 subfamily), Hybosoridae (2 species belonging to 1 subfamily). Dominance diversity graph show differences in the beetle's population of these 6 families (Fig. 1).

Table 1: An updated checklist of coleopteran families 2015

\begin{tabular}{|c|c|c|c|}
\hline S.No. & Name of species & Subfamily & Family \\
\hline 1. & Scarabaeus sanctus (Fabricius) & Scarabaeinae & Scarabaeidae \\
\hline 2. & Sisyphus neglectus Gory & Scarabaeinae & Scarabaeidae \\
\hline 3. & Sisyphus longipers (Olivier) & Scarabaeinae & Scarabaeidae \\
\hline 4. & Gymnopleurus cyaneus (Fabricius) & Scarabaeinae & Scarabaeidae \\
\hline 5. & Gymnopleurus gemmatus Harold & Scarabaeinae & Scarabaeidae \\
\hline 6. & Gymnopleurus miliaris (fabricius) & Scarabaeinae & Scarabaeidae \\
\hline 7. & Paragymnopleurus sinuatus (Olivier) & Scarabaeinae & Scarabaeidae \\
\hline 8. & Garreta smaragdifer (Walker) & Scarabaeinae & Scarabaeidae \\
\hline 9. & Garreta dejeani (Castelnau) & Scarabaeinae & Scarabaeidae \\
\hline 10. & Garreta mundus (Wiedemann) & Scarabaeinae & Scarabaeidae \\
\hline 11. & Heliocopris bucephalus (Fabricius) & Scarabaeinae & Scarabaeidae \\
\hline 12. & Catharsius pithecius (fabricius) & Scarabaeinae & Scarabaeidae \\
\hline 13. & Catharsius molossus (Linnaeus) & Scarabaeinae & Scarabaeidae \\
\hline 14. & Catharsius sagax (Quenstedt) & Scarabaeinae & Scarabaeidae \\
\hline 15. & Caccobius unicornis (Fabricius) & Scarabaeinae & Scarabaeidae \\
\hline 16. & Copris carinicus Gillet & Scarabaeinae & Scarabaeidae \\
\hline 17. & Copris repertus Walker & Scarabaeinae & Scarabaeidae \\
\hline 18. & Copris imitans Felsche & Scarabaeinae & Scarabaeidae \\
\hline 19. & Copris surdus Arrow & Scarabaeinae & Scarabaeidae \\
\hline 20. & Phalops divisus (Wiedemann) & Scarabaeinae & Scarabaeidae \\
\hline 21. & Onthophagus igneus Vigors & Scarabaeinae & Scarabaeidae \\
\hline 22. & Onthophagus pactolus (Fabricius) & Scarabaeinae & Scarabaeidae \\
\hline 23. & Onthophagus cervus (Fabricius) & Scarabaeinae & Scarabaeidae \\
\hline 24. & Onthophagus ramosellus Bates & Scarabaeinae & Scarabaeidae \\
\hline 25. & Onthophagus bonasus (Fabricius) & Scarabaeinae & Scarabaeidae \\
\hline 26. & Onthophagus griseosetosus Arrow & Scarabaeinae & Scarabaeidae \\
\hline 27. & Onthophagus gazella (Fabricius) & Scarabaeinae & Scarabaeidae \\
\hline 28. & Onthophagus ramosus (Wiedemann) & Scarabaeinae & Scarabaeidae \\
\hline 29. & Onthophagus spinifex (Fabricius) & Scarabaeinae & Scarabaeidae \\
\hline 30. & Onthophagus dama (Fabricius) & Scarabaeinae & Scarabaeidae \\
\hline 31. & Onthophagus quadridentatus (Fabricius) & Scarabaeinae & Scarabaeidae \\
\hline 32. & Onthophagus armatus Blanchard & Scarabaeinae & Scarabaeidae \\
\hline 33. & Onthophagus agnus Gillet & Scarabaeinae & Scarabaeidae \\
\hline 34. & Onthophagus ludio Boucomon & Scarabaeinae & Scarabaeidae \\
\hline 35. & Onthophagus hindu Arrow & Scarabaeinae & Scarabaeidae \\
\hline 36. & Onthophagus abreui Arrow & Scarabaeinae & Scarabaeidae \\
\hline 37. & Onthophagus unifasciatus (Schaller) & Scarabaeinae & Scarabaeidae \\
\hline 38. & Onthophagus Sagittarius (Fabricius) & Scarabaeinae & Scarabaeidae \\
\hline 39. & Onitis philemon Fabricius & Scarabaeinae & Scarabaeidae \\
\hline 40. & Onitis subopacus Arrow & Scarabaeinae & Scarabaeidae \\
\hline 41. & Oniticellus cinctus (Fabricius) & Scarabaeinae & Scarabaeidae \\
\hline 42. & Paragymnopleurus sinuatus (Olivier) & Scarabaeinae & Scarabaeidae \\
\hline 43. & Tiniocellus spinipes (Roth) & Scarabaeinae & Scarabaeidae \\
\hline 44. & Tibiodrepanus setosus (Wiedemann) & Scarabaeinae & Scarabaeidae \\
\hline 45. & Drepanocerus setosus (Wiedemann) & Scarabaeinae & Scarabaeidae \\
\hline 46. & Anomala bengalensis (Blanchard) & Rutelinae & Scarabaeidae \\
\hline 47. & Anomala biharensis Arrow & Rutelinae & Scarabaeidae \\
\hline 48. & Anomala cantori (Hope) & Rutelinae & Scarabaeidae \\
\hline 49. & Anomala dorsalis (Fabricius) & Rutelinae & Scarabaeidae \\
\hline 50. & Anomala polita (Blanchard) & Rutelinae & Scarabaeidae \\
\hline 51. & Anomala ruficapilla Burmeister & Rutelinae & Scarabaeidae \\
\hline 52. & Anomala rugosa Arrow & Rutelinae & Scarabaeidae \\
\hline 53. & Anomala varicolor (Gyllenhal) & Rutelinae & Scarabaeidae \\
\hline 54. & Anomala sp. & Rutelinae & Scarabaeidae \\
\hline 55. & Mimela macleayana (Vigors) & Rutelinae & Scarabaeidae \\
\hline
\end{tabular}




\section{International Journal of Science and Research (IJSR) ISSN (Online): 2319-7064}

Index Copernicus Value (2013): 6.14 | Impact Factor (2015): 6.391

\begin{tabular}{|c|c|c|c|}
\hline 56. & Mimela inscripta (Nonfried) & Rutelinae & Scarabaeidae \\
\hline 57. & Popillia laevis Burmeister & Rutelinae & Scarabaeidae \\
\hline 58. & Adoretus lasiopygus Burmeister & Rutelinae & Scarabaeidae \\
\hline 59. & Adoretus bicolor Brenske & Rutelinae & Scarabaeidae \\
\hline 60. & Adoretus limbatus Blanchard & Rutelinae & Scarabaeidae \\
\hline 61. & Adoretus bimarginatus Ohaus & Rutelinae & Scarabaeidae \\
\hline 62. & Adorectus duvauceli Blanchard & Rutelinae & Scarabaeidae \\
\hline 63. & Xylotrupes gideon (Linnaeus) & Dynastinae & Scarabaeidae \\
\hline 64. & Alissonotum simile Arrow & Dynastinae & Scarabaeidae \\
\hline 65. & Phyllognathus dionysius (Fabricius) & Dynastinae & Scarabaeidae \\
\hline 66. & Heteronychus lioderes Redtenbacher & Dynastinae & Scarabaeidae \\
\hline 67. & Eophileurus platypterus (Wiedmann) & Dynastinae & Scarabaeidae \\
\hline 68. & Apogonia proxima Waterhouse & Melolonthinae & Scarabaeidae \\
\hline 69. & Schizonycha ruficollis (Fabricius) & Melolonthinae & Scarabaeidae \\
\hline 70. & Holotrichia sculpticollis Blanchard & Melolonthinae & Scarabaeidae \\
\hline 71. & Holotrichia problemetica Brenske & Melolonthinae & Scarabaeidae \\
\hline 72. & Clinteria klugi (Hope) & Cetoniinae & Scarabaeidae \\
\hline 73. & Gametis versicolor (Fabricius) & Cetoniinae & Scarabaeidae \\
\hline 74. & Orphnus impressus Westwood & Orphninae & Scarabaeidae \\
\hline 75. & Orphnus parvus (Wiedemann) & Orphninae & Scarabaeidae \\
\hline 76. & Aphodius moestus Fabricius & Aphodiinae & Scarabaeidae \\
\hline 77. & Aphodius crenatus Harold & Aphodiinae & Scarabaeidae \\
\hline 78. & Rhyssemus germanus (Linnaeus) & Aphodiinae & Scarabaeidae \\
\hline 79. & Hybosorus orientalis Westwood & Hybosorinae & Hybosoridae \\
\hline 80. & Phaeochrous emarginatus (Castelnau) & Hybosorinae & Hybosoridae \\
\hline 81. & Bolbohamatum marginale Krikken & Bolboceratinae & Geotrupidae \\
\hline 82. & Bolbohamatum calanus (Westwood) & Bolboceratinae & Geotrupidae \\
\hline 83. & Bolbohamatum phallosum Krikken & Bolboceratinae & Geotrupidae \\
\hline 84. & Bolboceras quadridens (Fabricius) & Bolboceratinae & Geotrupidae \\
\hline 85. & Prionomma atratum (Gmelin) & Prioninae & Cerambycidae \\
\hline 86. & Bandar pascoei pascoei (Lansberge) & $\begin{array}{c}\text { Prioninae } \\
\end{array}$ & Cerambycidae \\
\hline 87. & Acanthophorous serraticornis (Olivier) & Prioninae & Cerambycidae \\
\hline 88. & Lema coromandeliana Fabricius & Criocerinae & Chrysomelidae \\
\hline 89. & Lema $($ Oulema $)$ downesi Baly & Criocerinae & Chrysomelidae \\
\hline 90. & Lema histrio Clark & Criocerinae & Chrysomelidae \\
\hline 91. & Lema maheensis Jacoby & Criocerinae & Chrysomelidae \\
\hline 92. & Lema praeusta Fabricius & Criocerinae & Chrysomelidae \\
\hline 93. & Lema rufotestacea Clark & Criocerinae & Chrysomelidae \\
\hline 94. & Lema semifulva Jacoby & Criocerinae & Chrysomelidae \\
\hline 95. & Lema singularis Jacoby & Criocerinae & Chrysomelidae \\
\hline 96. & Lema terminate Lacordaire & Criocerinae & Chrysomelidae \\
\hline 97. & Lema tibiella Weise & Criocerinae & Chrysomelidae \\
\hline 98. & Gynandrophthalma longicornis Jacoby & Clytrinae & Chrysomelidae \\
\hline 99. & Gynandrophthalma divisa Jacoby & Clytrinae & Chrysomelidae \\
\hline 100. & Gynandrophthalma duvivieri Jacoby & Clytrinae & Chrysomelidae \\
\hline 101. & Aetheomorpha maduraensis Jacoby & Clytrinae & Chrysomelidae \\
\hline 102. & Aetheomorpha fallax Lacordaire & Clytrinae & Chrysomelidae \\
\hline 103. & Aspidolopha sp. & Clytrinae & Chrysomelidae \\
\hline 104. & Cryptocephalus bissexsignatus Suffrian & Cryptocephalinae & Chrysomelidae \\
\hline 105. & Cryptocephalus ovulum Suffrian & Cryptocephalinae & Chrysomelidae \\
\hline 106. & Cryptocephalus pallidipennis Jacoby & Cryptocephalinae & Chrysomelidae \\
\hline 107. & Cryptocephalus sehestedti Fabricius & Cryptocephalinae & Chrysomelidae \\
\hline 108. & Cryptocephalus sexsignatus Fabricius & Cryptocephalinae & Chrysomelidae \\
\hline 109. & Cryptocephalus uniformis Jacoby & Cryptocephalinae & Chrysomelidae \\
\hline 110. & Cryptocephalus vahli Fabricius & Cryptocephalinae & Chrysomelidae \\
\hline 111. & Cryptocephalus sp. & Cryptocephalinae & Chrysomelidae \\
\hline 112. & Chlamys stercoralis Gressitt & Chlamisinae & Chrysomelidae \\
\hline 113. & Abirus sp. & Eumolpinae & Chrysomelidae \\
\hline 114. & Basilepta latefaciata Jacoby & Eumolpinae & Chrysomelidae \\
\hline 115. & Colasposoma auripenne Motschulsky & Eumolpinae & Chrysomelidae \\
\hline 116. & Pachnephorus impressus Rosenh & Eumolpinae & Chrysomelidae \\
\hline 117. & Pagria signata Motschulsky & Eumolpinae & Chrysomelidae \\
\hline 118. & Platycorynus peregrinus Fuessly & Eumolpinae & Chrysomelidae \\
\hline 119. & Chrysomela exanthematica Weidemann & Chrysomelinae & Chrysomelidae \\
\hline 120. & Chrysolina sp1 & Chrysomelinae & Chrysomelidae \\
\hline 121. & Chrysolina sp2. & Chrysomelinae & Chrysomelidae \\
\hline 122. & Aulacophora foveicollis Lucas & Galerucinae & Chrysomelidae \\
\hline
\end{tabular}

Volume 5 Issue 4, April 2016 


\section{International Journal of Science and Research (IJSR) ISSN (Online): 2319-7064}

Index Copernicus Value (2013): 6.14 | Impact Factor (2015): 6.391

\begin{tabular}{|c|c|c|c|}
\hline 123. & Aulacophora intermedia Jacoby & Galerucinae & Chrysomelidae \\
\hline 124. & Oides bipunctata Fabricius & Galerucinae & Chrysomelidae \\
\hline 125. & Madurasia obscurella Jacoby & Galerucinae & Chrysomelidae \\
\hline 126. & Medythia suturalis Motschulsky & Galerucinae & Chrysomelidae \\
\hline 127. & Monolepta bifasciata Hornstedt & Galerucinae & Chrysomelidae \\
\hline 128. & Monolepta brunnea Maulik & Galerucinae & Chrysomelidae \\
\hline 129. & Monolepta conformis Weise & Galerucinae & Chrysomelidae \\
\hline 130. & Monolepta lineata Weise & Galerucinae & Chrysomelidae \\
\hline 131. & Monolepta marginipennis Jacoby & Galerucinae & Chrysomelidae \\
\hline 132. & Monolepta nigrobilineata Motschulsky & Galerucinae & Chrysomelidae \\
\hline 133. & Monolepta severini Jacoby & Galerucinae & Chrysomelidae \\
\hline 134. & Monolepta signata Olivier & Galerucinae & Chrysomelidae \\
\hline 135. & Monolepta sodalis Weise & Galerucinae & Chrysomelidae \\
\hline 136. & Sphenoraia bicolor Hope & Galerucinae & Chrysomelidae \\
\hline 137. & Aphthona hugely Weise & Alticinae & Chrysomelidae \\
\hline 138. & Aphthona kanaraensis Jacoby & Alticinae & Chrysomelidae \\
\hline 139. & Aphthona nigrilabris Duvivier & Alticinae & Chrysomelidae \\
\hline 140. & Chaetocnema basalis Baly & Alticinae & Chrysomelidae \\
\hline 141. & Chaetocnema bretinghami Baly & Alticinae & Chrysomelidae \\
\hline 142. & Chaetocnema concinnipennis Baly & Alticinae & Chrysomelidae \\
\hline 143. & Chaetocnema confines Crotch & Alticinae & Chrysomelidae \\
\hline 144. & Chaetocnema harita Maulik & Alticinae & Chrysomelidae \\
\hline 145. & Crepidodera minuta Jacoby & Alticinae & Chrysomelidae \\
\hline 146. & Crepidodera nigripennis Motschulsky & Alticinae & Chrysomelidae \\
\hline 147. & Haltica cyanea Weber & Alticinae & Chrysomelidae \\
\hline 148. & Hermacophaga ruficollis Lucas & Alticinae & Chrysomelidae \\
\hline 149. & Hyphasis $\mathrm{sp}$ & Alticinae & Chrysomelidae \\
\hline 150. & Longitarsus lohita Maulik & Alticinae & Chrysomelidae \\
\hline 151. & Longitarsus pandura Maulik & Alticinae & Chrysomelidae \\
\hline 152. & Longitarsus recticolis Maulik & Alticinae & Chrysomelidae \\
\hline 153. & Philopona signata Duvivier & Alticinae & Chrysomelidae \\
\hline 154. & Phygasia hookeri Baly & Alticinae & Chrysomelidae \\
\hline 155. & Phygasia unicolor Olivier & Alticinae & Chrysomelidae \\
\hline 156. & Phygasia violaceipennis Jacoby & Alticinae & Chrysomelidae \\
\hline 157. & Phyllotreta birmanica Harold & Alticinae & Chrysomelidae \\
\hline 158. & Phyllotreta chotanica Duvivier & Alticinae & Chrysomelidae \\
\hline 159. & Podagrica nigripennis Jacoby & Alticinae & Chrysomelidae \\
\hline 160. & Choeridina picea Baly & Hispinae & Chrysomelidae \\
\hline 161. & Dactylispa pusilal Weise & Hispinae & Chrysomelidae \\
\hline 162. & Oncocephala quadrilobata Guerin & Hispinae & Chrysomelidae \\
\hline 163. & Platypria andrewesi Weise & Hispinae & Chrysomelidae \\
\hline 164. & Aspidimorpha furcata Thunberg & Cassidinae & Chrysomelidae \\
\hline 165. & Aspidimorpha lobata Boheman & Cassidinae & Chrysomelidae \\
\hline 166. & Aspidimorpha miliaris Fabricius & Cassidinae & Chrysomelidae \\
\hline 167. & Aspidimorpha sanctaecrucis Fabricius & Cassidinae & Chrysomelidae \\
\hline 168. & Cassida circumdata Herbst & Cassidinae & Chrysomelidae \\
\hline 169. & Conchyloctenia nigrovittata Boheman $\cup$ & Cassidinae & Chrysomelidae \\
\hline 170. & Cassida circumdata Herbst & Cassidinae & Chrysomelidae \\
\hline 171. & Cassida exilis Boheman & Cassidinae & Chrysomelidae \\
\hline 172. & Cassida nilgiriensis Borowiec & Cassidinae & Chrysomelidae \\
\hline 173. & Cassida obtusata Boheman & Cassidinae & Chrysomelidae \\
\hline 174. & Cassida pulvinata Boheman & Cassidinae & Chrysomelidae \\
\hline 175. & Cassida residua Weise & Cassidinae & Chrysomelidae \\
\hline 176. & Chiridopsis bipunctata Linnaeus & Cassidinae & Chrysomelidae \\
\hline 177. & Chiridopsis sp.1 & Cassidinae & Chrysomelidae \\
\hline 178. & Chiridopsis nigropunctata Borowiec & Cassidinae & Chrysomelidae \\
\hline 179. & Chiridopsis sp.2 & Cassidinae & Chrysomelidae \\
\hline 180. & Glyhocasis trilineata Hope & Cassidinae & Chrysomelidae \\
\hline 181. & Oocassida pudibunda Boheman & Cassidinae & Chrysomelidae \\
\hline 182. & Rhytidocassis indicola Duvivier & Cassidinae & Chrysomelidae \\
\hline 183. & Luprops gracillior Fairmaire & Lagriinae & Tenebrionidae \\
\hline 184. & Playnotus punctatipennis Mulsant & Tenebrioninae & Tenebrionidae \\
\hline 185. & Gonocephalum catenulatum (Fairmaire) & Tenebrioninae & Tenebrionidae \\
\hline 186. & Gonocephalum tuberculatum (Hope) & Tenebrioninae & Tenebrionidae \\
\hline 187. & Heterotarsus crenatus Fairmaire & Tenebrioninae & Tenebrionidae \\
\hline 188. & Heterotarsus foveoseriatus Fairmaire & Tenebrioninae & Tenebrionidae \\
\hline 189. & Mesomorphus villager Blanch & Tenebrioninae & Tenebrionidae \\
\hline
\end{tabular}

\section{Volume 5 Issue 4, April 2016}




\section{International Journal of Science and Research (IJSR) \\ ISSN (Online): 2319-7064}

Index Copernicus Value (2013): 6.14 | Impact Factor (2015): 6.391

\begin{tabular}{|l|c|c|c|}
\hline 190. & Scleron bengalensis Kaszab & Tenebrioninae & Tenebrionidae \\
\hline 191. & Scleron latipes Guerin & Tenebrioninae & Tenebrionidae \\
\hline 192. & Anacycus navicularis Fairmaire & Tenebrioninae & Tenebrionidae \\
\hline 193. & Derosphaerus cribrum Fairmaire & Stenochiinae & Tenebrionidae \\
\hline 194. & Promethis impressa (Fabricius) & Stenochiinae & Tenebrionidae \\
\hline
\end{tabular}

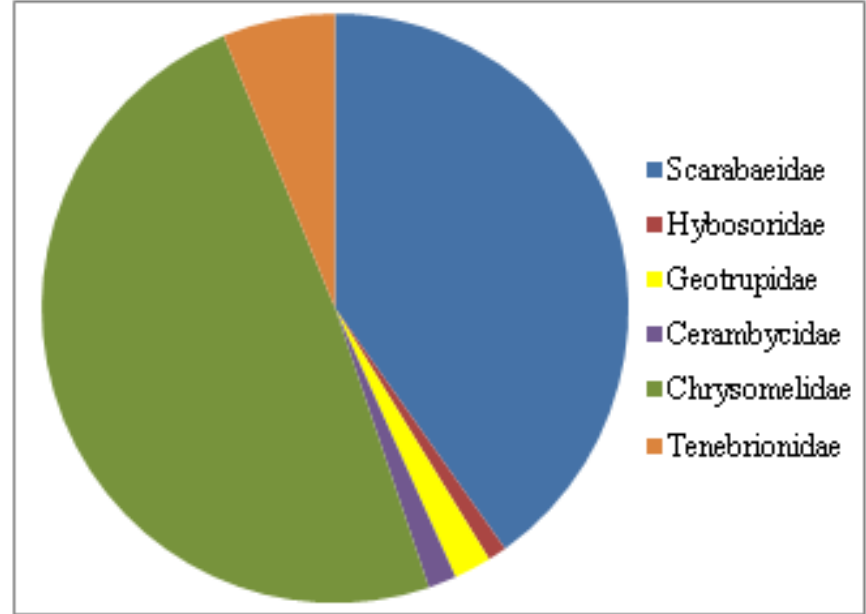

Figure 1: Family distribution of beetles in the Chhattisgarh state

\section{Acknowledgement}

The authors are thankful to the Head of the Department, School of Studies in Bioscience (Life Sciences) and UGC New Delhi for their financial support as BSR Fellowship to one of us (NG).

\section{References}

[1] Banerjee, M. (2014). Diversity and Composition of Beetles (Order: Coleoptera) of Durgapur,West Bengal, India. Psyche: A Journal of Entomology, 1-3.

[2] Chandra, K., and Gupta, D. (2012). Bolboceratine Scarab of genera Bolbohamatum Krikken, 1980 and Bolbogonium Boucomont, 1911 (Coleoptera:Geotrupidae) from central India. Taprobanica: The Journal of Asian Biodiversity, 4, 6976.

[3] Chandra, K., and Gupta, D. (2012a). An inventory of Scarab Beetles (Coleoptetra: Scarabaeidae) of Achanakmar-Amarkantak Biosphere Reserve, Chhattisgarh, India. International Journal of Science and Nature, 3, 886-891.

[4] Chandra, K., and Gupta, D. (2012b). Diversity and Relative Abundance of Pleurostict Scarabaeidae (Coleoptera) in Achanakmar- Amarkantak Biosphere Reserve, Central India. World Journal of Zoology, 7, 147-154.

[5] Chandra, K., and Gupta, D. (2013a). Scarab Beetles (Coleoptera: Scarabaeoidea) of Barnawapara Wildlife Sanctuary, Chhattisgarh, India. Journal of Threatened Taxa, 5, 4660-4671.

[6] Chandra, K., and Gupta, D. (2013b). Taxonomic Studies on Dung Beetles (Coleoptera: Scarabaeidae, Geotrupidae, Hybosoridae) of Chhattisgarh, India. Munis Entomology and Zoology, 8, 331-360.
[7] Choate, P. M. (2001). Manual for identification of ground beetles (Coleoptera: Carabidae) (including tiger beetles) of Florida. 1-19.

[8] Hegde, V. D., Lal, B., and Chandra, K. (2015). New records of darkling beetles (Tenebrionidae: Coleoptera) from Chhattisgarh, India. Biological Forum- An International journal. 7, 707-711.

[9] Kalaichelvan, T., and Verma, K. K. (2005a). Life History and Biology of Lema Semifulva Jac. (Coleoptera: Chrysomelidae: Criocerinae) at BhilaiDurg. Zoos 'Print Journal, 20, 1896-1898.

[10] Kalaichelvan, T., and Verma, K. K. (2005b). Checklist of Leaf Beetles (Coleoptera: Chrysomelidae) of BhilaiDurg. Zoos' Print Journal, 20, 1838-1842.

[11]Kazmi, S. I., and Ramamurthy, V. V. (2004). Coleoptera (Insect) fauna from the Indian Thar Desert, Rajasthan. Zoos' Print Journal, 19, 1447-1448.

[12] Thakare,V., Zade, V., Chandra, K., and Gupta, D. (2012). Scarab Beetle (Coleoptera: Scarabaeoidea) of Melghat Tiger Reserve, Central India. Journal of Entomology, 5, 73-80.

[13] Pawara, R. H., Patel, N.G., Pawara, J.V., Gavit, P. J., and Ishi, S.S. (2014). Beetles of Jalgaon District of Maharashtra, India. Biolife, 2, 970-973.

\section{Authors Profile}

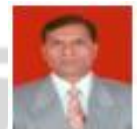

Dr. Shiv Kant Prasad, Ph.D; MZS is Professor. He did Ph.D in Zoology (1992) - Banaras Hindu University. UGC-JRF/SRF, CSIR-SRF/Research Associate- Banaras Hindu University, Lecturer/Assistant Professor (1996-2005); Reader/Associate Professor (2005-2009) -School of Studies in Zoology and Biotechnology, Vikram University, Ujjain (M.P) Professor (2009Present)-School of Studies in Bioscience (Life Science), Pt. Ravishankar Shukla University, Raipur (C.G). Recipient of UGC Research Award, 2004. He is recipient of Best Scientific Research Publication Award (2008-2009) - M.P. Council of Science and Technology, Bhopal. His specialization includes Zoology/ Endocrinology and Reproductive physiology

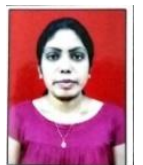

Namita Gajendra, M. Sc, MPhil is Research scholar, Zoology School of studies in Biosciense (Life Science) Pt Ravishankar Shukla University, Raipur, Chhattisgarh, 492001 